\title{
Survival probabilities in time-dependent random walks
}

\author{
Ehud Nakar and Shahar Hod \\ The Racah Institute of Physics, The Hebrew University, Jerusalem 91904, Israel
}

(December 22, 2018)

\begin{abstract}
We analyze the dynamics of random walks in which the jumping probabilities are periodic timedependent functions. In particular, we determine the survival probability of biased walkers who are drifted towards an absorbing boundary. The typical life-time of the walkers is found to decrease with an increment of the oscillation amplitude of the jumping probabilities. We discuss the applicability of the results in the context of complex adaptive systems.
\end{abstract}

Random walk is one of the most ubiquitous concepts of statistical physics. In fact, it finds applications in virtually every area of physics (see e.g., [1-6] and references therein). Random walks in the presence of absorbing traps are much studied in recent years as models for absorbing-state phase transitions $[7,8]$, polymer adsorption [9], granular segregation [10], and the spreading of an epidemic [11].

One of the main characteristics of a random walk in the presence of an absorbing boundary is the survival probability $S\left(t_{0}\right)$ of the the walkers, the probability that a walker has not reached the absorption point before $t_{0}$. When the walkers are drifted towards the absorbing point, the survival probability falls exponentially at asymptotically late-times $S(t) \propto \exp \left(-t / \tau_{s}\right)$, where the characteristic life-time $\tau_{s}$ depends on the drift velocity (see e.g. [12]).

The problem of random walks with an absorption boundary has recently been extended to include situation in which the jumping probabilities of the walkers are time-dependent [13]. The solution presented in [13] accounts for the binary (alternating) case, namely $p(t)=\frac{1}{2}-\epsilon_{p}+(-1)^{t} A$, where $p(t)$ is the probability to step to the right at time $t$. Here $A$ is the oscillations amplitude, and $\epsilon_{p}>0$ represents a net drift towards the absorbing boundary (the period of such binary oscillations is $T=2$ ). It was found [13] that the characteristic life-time of the walkers $\tau_{s}$ depends on the amplitude $A$, in a non-trivial way. In particular, $\tau_{s}$ was shown to decrease monotonically with the increment of $A$. While this analysis provides a useful insight into the behavior of such time-dependent random walks, it is of one's interest to explore the general case time-dependent jumping probabilities with $T \neq 2$. This is the main goal of the present work.

In addition to the intrinsic interest in such timedependent random walks, our work may find direct applications in many complex physical, biological, and economical systems. In fact, the main motivation for the introduction of time-dependent random walks in [13] was its applicability in the flourishing field of complex adaptive systems. In the well-studied model of the minority game (MG) [14], and its evolutionary version (EMG) [15] (see also [16-28]), it was found that the winning probabilities of the agents display a periodic behavior in time. This implies that the survival probabilities of the agents are well-described by a model of a periodic time-dependent random walk with an absorbing boundary [13]. The analytical model presented in [13] (with $T=2$ ) provides an elegant explanation for the intriguing phase-transition observed in the EMG [25] (from selfsegregation to clustering as the prize-to-fine ratio drops below some critical value). However, numerical studies of the EMG [27] have indicated that the period of the oscillations (in the winning probabilities of the agents) depends on the specific parameters of the system. Extending the analysis of [13] to the general case of timedependent jumping probabilities with $T \neq 2$ is therefore highly motivated.

We consider a time dependent random walker who is drifted towards an absorption boundary, located at $-d$ (where $d>0$ ). The drift towards the absorption point may be attributed to two distinct reasons: (i) A smaller (average) probability to take a step to the right (away from the absorption point). That is, $\langle p(t)\rangle=1 / 2-\epsilon_{p}$ where $\epsilon_{p}>0$, and (ii) a smaller step size $r$ to the right, $0 \leq r<1$ (where the step size to the left is scaled to $1)$. This kind of drift is characterized by the parameter $\epsilon_{r} \equiv 1 /(1+r)-\frac{1}{2}$. In the unbiased case $\left(\epsilon_{p}=\epsilon_{r}=0\right)$ there is no net drift, and the survival probability is well known to scale as an inverse power-law: $S(t) \propto t^{-1 / 2}$. In the presence of a drift $\left(\epsilon_{p}>0\right.$ and/or $\left.\epsilon_{r}>0\right)$, and with a constant (time-independent) jumping probabilities, one finds (see e.g. [12])

$$
S(t) \propto t^{-\frac{3}{2}} e^{-t / \tau_{s}} .
$$

This result holds true in the binary time-dependent model [13] as well, in which case the jumping probability is given by the alternating function $p(t)=\frac{1}{2}-\epsilon_{p}+(-1)^{t} A$.

Below we explore the time-dependence of the survival probability $S(t)$ for a general periodic $p(t)$. Let

$$
p(t)=\frac{1}{2}+F(t)
$$

where $F(t)$ is a periodic function, with a period $T$. The mean of $F$ (over one period) is given by 


$$
\langle F\rangle \equiv(1 / T) \sum_{i=1}^{T} F(i)=-\epsilon_{p} .
$$

In the time-independent case it has been established that the probability $B(t)$ for a random walker (with no absorbing boundary) to be located on the right hand side $[x(t)>0]$ at the time $t$ is given by $B(t) \propto \exp \left[-t / \tau_{B}\right]$, with $\tau_{B}=\tau_{s}$ (see e.g., [12]). Motivated by the equality $\tau_{B}=\tau_{s}$ in the time independent case, we will find the asymptotic form of $\tau_{B}$ in the general case of timedependent jumping probabilities. We will establish the relation $\tau_{B}(T=2) \approx \tau_{s}(T=2)$ analytically, and demonstrate numerically that $\tau_{B}(T \neq 2) \approx \tau_{s}(T \neq 2)$.

We first consider the case in which the period $T$ of the oscillations is a natural number. Let $\omega(t)$ be the number of right steps taken by the walker out of $t$ steps. The walker is located at the right hand side $[x(t)>0]$ if $\omega>\omega_{c}$, where

$$
\omega_{c}=\frac{t-d}{1+r} \simeq t\left(\frac{1}{2}+\epsilon_{r}\right)
$$

in the $t \gg d, T$ limit.

Note that $\omega$ is a sum of $T$ independent binomial distributions with a success probability $p_{i}=p(t=i)$. After time $t \gg T$ a step is chosen $\simeq t / T$ times from each one of these distributions. Thus, for $t \gg T, \omega$ is distributed normally with an average of $\mu_{w}(t)=t\left(\frac{1}{2}-\epsilon_{p}\right)$, and a variance $\sigma_{\omega}^{2}=\frac{t}{T} \sum_{i=1}^{T} p_{i}\left(1-p_{i}\right)=t\left(1-4\left\langle F^{2}\right\rangle\right) / 4$, where

$$
\left\langle F^{2}\right\rangle \equiv(1 / T) \sum_{i=1}^{T} F^{2}(i) .
$$

Hence, $B(t)$ can be approximated by this normal distribution, with the condition $\omega(t)>\omega_{c}(t)$

$$
\begin{aligned}
& B(t)=\frac{1}{\sqrt{2 \pi \sigma^{2}}} \int_{w_{c}}^{\infty} \exp \left[-\frac{(x-\mu)^{2}}{2 \sigma^{2}}\right] d x \\
= & \frac{1}{\sqrt{\pi}} \int_{\sqrt{t / \tau_{B}}}^{\infty} e^{-x^{2}} d x=\frac{1}{2} \operatorname{erfc}\left(\sqrt{t / \tau_{B}}\right),
\end{aligned}
$$

where

$$
\tau_{B}=\frac{2 \sigma^{2} t}{\left(w_{c}-\mu\right)^{2}}=\frac{1-4\left\langle F^{2}\right\rangle}{2\left(\epsilon_{p}+\epsilon_{r}\right)^{2}},
$$

and $\operatorname{erfc}$ is the complementary error function. The complementary error function can be approximated as $\operatorname{erfc}\left(\sqrt{t / \tau_{B}}\right) \simeq e^{-t / \tau_{B}} / \sqrt{\pi t / \tau_{B}}$ at asymptotically large times. One therefore finds

$$
B(t) \propto e^{-t / \tau_{B}}
$$

The model of binary time-dependent jumping probabilities $\left[F(t)=-\epsilon_{p}+A(-1)^{t}\right]$ was solved exactly in [13], the expression for $\tau_{s}$ is given by Eq. (16) of [13]. Expanding this expression for small $\epsilon_{p}$ and $\epsilon_{r}$, one finds

$$
\tau_{s}(T=2) \simeq \frac{1-4 A^{2}}{2\left(\epsilon_{p}+\epsilon_{r}\right)^{2}} .
$$

This agrees with Eq. (7) in the limit $\epsilon_{p} \ll 1$ (or equivalently, $\left.\tau_{s} \gg 1\right)$. One therefore finds $\tau_{s}(T=2) \simeq \tau_{B}(T=$ 2) in the $\tau_{s} \gg 1$ limit.

The generalization of the above analysis for an arbitrary period $T$ is straightforward. If $T=m / n$ is a rational number, then $F(t)$ could be replaced by an equivalent function with a period $m$ which is a natural number. If $T$ is an irrational, then the all range of values of $F(0<t<T)$ is sampled (over many periods of the oscillations). In this case, one should replace Eqs. (3) and (5) by

$$
\langle F\rangle=\frac{1}{T} \int_{0}^{T} F(t) d t=-\epsilon,
$$

and

$$
\left\langle F^{2}\right\rangle=\frac{1}{T} \int_{0}^{T} F^{2}(t) d t .
$$

In order to confirm the analytical predictions (in the $T \neq 2$ case), we perform numerical simulations of (discrete) random walks. We first consider the case of an harmonic function $p(t)$ of the form

$$
p(t)=\frac{1}{2}-\epsilon_{p}-A \cos \left(\frac{2 \pi t}{T}\right) ; T>1 .
$$

In this case $\langle F\rangle=-\epsilon_{p}$, and

$$
\left\langle F^{2}\right\rangle=\frac{1}{4}\left\{\begin{array}{c}
\epsilon_{p}^{2}+A^{2} ; \quad T=2 \\
\epsilon_{p}^{2}+(A / \sqrt{2})^{2} ; \quad T \neq 2
\end{array}\right.
$$

This implies that for $T \neq 2, \tau_{B}$ is independent of the period $T$ of the oscillations. Moreover, we find that $\tau_{B}(T \neq 2, A / \sqrt{2})=\tau_{B}(T=2, A)$. As we know that our approximation holds true for $T=2$ (The exact shape of $F$ is irrelevant in this case), one should compare the survival probabilities in the $T=2$ and $T \neq 2$ cases. Figure 1 depicts $t\left(S=10^{-5}\right)$, the step number in which the survival probability has fallen to $S=10^{-5}$, as a function of the oscillation period $T$ (and for three different values of the oscillation amplitude $A$ ). As predicted by Eq. (13), the survival probabilities are almost independent of the period $T$, except in the unique case of binary oscillations with $T=2$. Moreover, $S(t ; T=2)<S(t ; T \neq 2)$, in agreement with Eqs. (7) and (13)]. In addition, the numerical simulations indicate that the survival probability decreases monotonically with an increment in the oscillation amplitude $A$, in agreement with the analytical prediction Eq. (7).

In Figure 2 we confirm the relation $S(t, T \neq 2, A)=$ $S(t, T=2, \sqrt{2} A)$ in the harmonic case. The figure displays the step number $t\left(S=10^{-6}\right)$ for which the survival probabilities have fallen to $10^{-6}$. It is clear that 


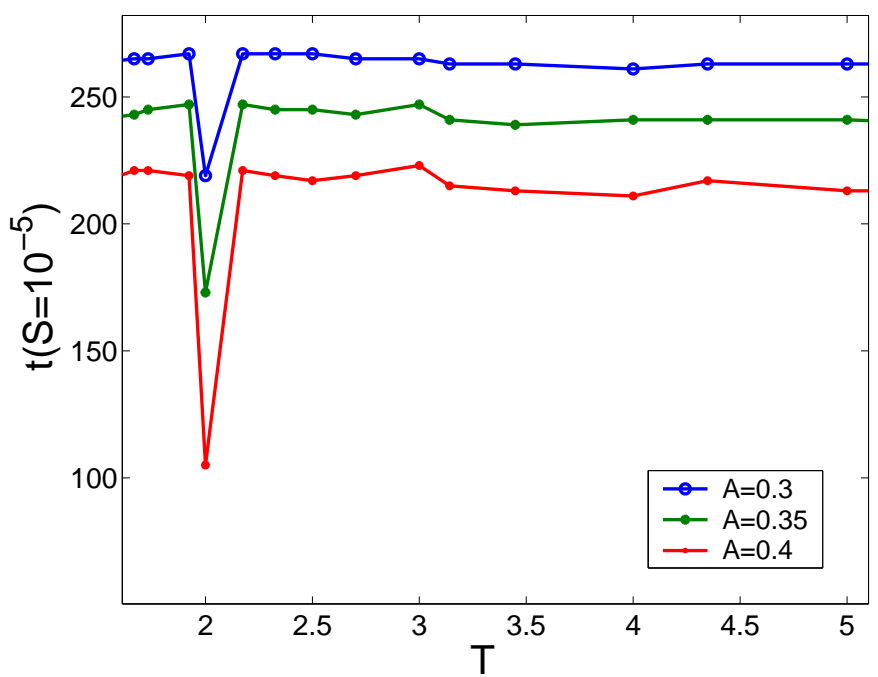

FIG. 1. The step number for which the survival probability has fallen to $S=10^{-5}$, as a function of the oscillation period $T$, and for three different values of $A$. The jumping probabilities $p(t)$ are characterize by harmonic oscillations, and are given by Eq. (12). We use $N=10^{8}$ walkers, $\epsilon_{p}=0.1, r=1$, and $d=2 . S(t)$ is independent of the oscillation period $T$, except in the unique case of binary oscillations with $T=2$ [in which case $S(t)$ is smaller, in agreement with the analytical prediction.] In addition, $S(t)$ decreases monotonically with the increment of the oscillations amplitude $A$.

$t\left(S=10^{-6}\right)$ for $T=2$ agrees with the corresponding value in the $T \neq 2$ case, provided one uses the transformation $A \rightarrow \sqrt{2} A$.

We further check the validity of the analytical results for $F(t)$ in the form of a square wave of amplitude $A$, and a mean value $-\epsilon_{p}$. In this case one finds $\left\langle F^{2}\right\rangle=\epsilon_{p}^{2}+A^{2}$ for any $T$. This implies that $\tau_{B}(T)$ should be independent of the period $T$ of the oscilations (and in particular, $\tau_{s}(T)=\tau_{s}(T=2)$, where $\tau_{s}(T=2)$ was derived analytically in [13]). Figure 3 depicts $t\left(S=10^{-6}\right)$, the step number for which the survival probability has fallen to $S=10^{-6}$, as a function of the period $T$. We display results for three different values of the oscillation amplitude $A$. The numerical results confirm the finding according to which $S(t ; T)$ is independent of the value of $T$. Furthermore, the survival probability $S(t ; T)$ decreases with increasing amplitude $A$, in agrrement with Eq. (7).

In summary, in this paper we studied the survival probabilities of biased (drifted) random walkers, whose jumping probabilities are time-dependent. This is an extension of the binary case (with $T=2$ ), studied in [13]. The long-time asymptotic survival probability is dominated by an exponential fall $S(t) \propto \exp \left[-t / \tau_{s}\right]$. We have found a simple approximation for $\tau_{s}$ [see Eq. (7)], which solely depends on the first and the second moments of the oscillations $\left(\langle F\rangle\right.$ and $\left\langle F^{2}\right\rangle$ ), and on the step-size drift parameter $\epsilon_{r}$.

Our analytical results imply that the characteristic lifetime of the walkers $\tau_{s}$ decreases with increasing $\left\langle F^{2}\right\rangle$.

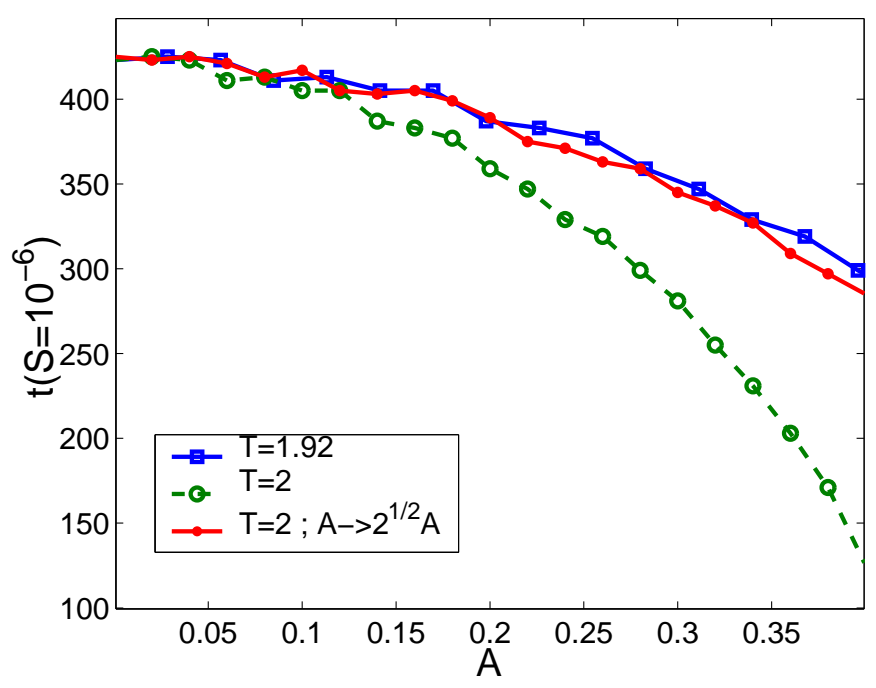

FIG. 2. The step number in which $S=10^{-6}$ as a function of the oscillations amplitude $A$, and for two different values of the period $T$. The results confirm the analytical prediction according to which $S(t, T=2, A)=S(t, T \neq 2, \sqrt{2} A)$, where $p(t)$ is an harmonic function. The parameters used are the same as in Fig. 1.

The larger are the temporal-oscillations in the jumping probabilities $p(t)$, the smaller is the survival probability. This result generalizes the one derived in [13] for the binary case (with $T=2$ ), in which case $\left\langle F^{2}\right\rangle=A^{2}+\epsilon_{p}^{2}$.

The qualitative nature of the anti-correlation between the survival probability $S(t)$ and the oscillation amplitude $\left\langle F^{2}\right\rangle$ is rather simple. It is a direct consequence of the positive correlation between $\sigma_{\omega}(t)$ (the dispersion in the access of right-steps taken out of $t$ steps) and $S(t)$ in the presence of a negative drift (towards the absorption boundary). In the presence of a net drift, $\mu_{\omega}$ (the average value of $\omega$ ) is smaller than $\omega_{c}$. A large $\sigma_{\omega}(t)$ is therefore required in order to survive, that is in order to have $\omega>\omega_{c}$ at asymptotically late-times [29].

Finally, the present analysis provides a direct explanation for the underlying mechanism responsible for the intriguing phase-transition observed in the evolutionary minority game $[25,15]$. In this model, the winning probabilities of the agents $p(t)$ where shown to display temporal oscillations [27]. The amplitude and period of these oscillations depend on the various parameters of the model (such as the price-to-fine ratio). In [13] a toymodel of time-dependent jumping probabilities with a period of $T=2$ was used to reveal the physical source for the phase-transition. In the present work we have shown that the anti-correlation between the characteristic lifetime $\tau_{s}$ and the oscillations amplitude $\left\langle F^{2}\right\rangle$ is a generic feature, independent of the period $T$ of the oscillations. Thus, our analysis (for generic values of $T$ ) lends support for the general applicability of the conclusions presented in [13]. 


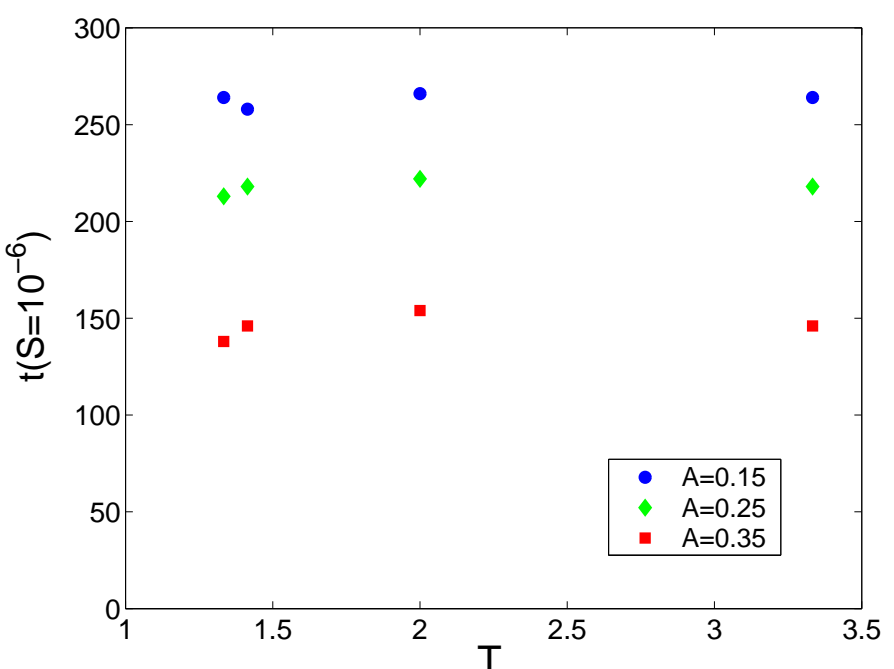

FIG. 3. The step number for which the survival probability has fallen to $S=10^{-6}$, as a function of the oscillation period $T$, and for three different values of $A . F(t)$ is a square wave with amplitude $A$, and a mean value $-\epsilon_{p}$. We use $N=10^{9}$ walkers, $\epsilon_{p}=0.1, R=0.9$, and $d=2$. We find that in this case, $S(t)$ is independent of the oscillation period $T$, in agreement with the analytical results.

\section{ACKNOWLEDGMENTS}

The research of SH was supported by G.I.F. Foundation. The research of EN was supported by the Horowitz foundation, and through the generosity of the Dan David Prize Scholarship 2003. We would also like to thank Yonatan Oren.

[1] M. N. Barber and B. W. Ninham, Random and Restricted Walks (Gordon and Breach, New York, 1970).

[2] N. G. van Kampen, Stochastic Processes in Physics and Chemistry (North-Holland, Amsterdam, 1992).

[3] R. Fernandez, J. Frohlich, and A. D. Sokal, Random Walks, Critical Phenomena, and Triviality in Quantum Field Theory (Springer Verlag, Berlin, 1992).

[4] G. H. Weiss, Aspects and Applications of the Random Walk (North Holland, Amsterdam, 1994).

[5] D. ben-Avraham and S. Havlin, Diffusion and Reactions in Fractals and Disordered Systems (Cambridge University Press, Cambridge, 2000).

[6] R. Dickman and D. ben-Avraham, Phys. Rev. E. 64, 020102(R) (2001).

[7] J. Marro and R. Dickman, Nonequilibrium Phase Transitions in Lattice Models, (Cambridge University Press, Cambridge, 1999).

[8] H. Hinrichsen, Adv. Phys. 49, 815 (2000).

[9] K. De'Bell and T. Lookman, Rev. Mod. Phys. 65, 87 (1993).
[10] Z. Farkasa and T. Fulop, J. Phys. A: Math. Gen. 34, 3191 (2001).

[11] P. Grassberger, H. Chate, and G. Rousseau, Phys. Rev. E 55, 2488 (1997).

[12] M. Bauer, C. Godreche and J. M. Luck, J. Stat. Phys. 96, 963 (1999).

[13] S. Hod, Phys. Rev. Lett. 90, 128701 (2003).

[14] D. Challet and C. Zhang, Physica A 246, 407 (1997); 256, 514 (1998); 269, 30 (1999).

[15] N. F. Johnson, P. M. Hui, R. Jonson, and T. S. Lo, Phys. Rev. Lett. 82, 3360 (1999).

[16] R. D‘Hulst and G. J. Rodgers, Physica A 270, 514 (1999).

[17] E. Burgos and H Ceva, Physica 284A, 489 (2000).

[18] T. S. Lo, P. M. Hui and N. F. Johnson, Phys. Rev. E 62, 4393 (2000).

[19] P. M. Hui, T. S. Lo, and N. F. Johnson, e-print condmat/0003309.

[20] M. Hart, P. Jefferies, N. F. Johnson and P. M. Hui, eprint cond-mat/0003486; e-print cond-mat/0004063.

[21] E. Burgos, H. Ceva and R. P. J. Perazzo, e-print condmat/0007010.

[22] T. S. Lo, S. W. Lim, P. M. Hui and N. F. Johnson, Physica 287A, 313 (2000).

[23] Y. Li, A. VanDeemen and R. Savit, e-print nlin.AO/0002004.

[24] R. Savit, R. Manuca and R. Riolo, Phys. Rev. Lett. 82, 2203 (1999).

[25] S. Hod and E. Nakar, Phys. Rev. Lett. 88, 238702 (2002).

[26] A. C. C. Coolen, e-print cond-mat/0205262.

[27] E. Nakar and S. Hod, Phys. Rev. E 67, 016109 (2003).

[28] I. Caridi and H. Ceva, e-print cond-mat/0206515.

[29] We note that in the unbiased case (with no drift), one finds $\mu_{\omega}>\omega_{c}$. A small $\sigma_{\omega}(t)$ therefore implies large survival probability. Thus, $\left\langle F^{2}\right\rangle$ is positively correlated with $S(t)$ in the unbiased case, in agreement with the results of [13] for the binary case. 